\title{
Photonic Quasi-Crystal LEDs: Design, modelling, and optimisation.
}

\author{
M.D.B. Charlton ${ }^{*}$, M.E. Zoorob ${ }^{\dagger}$, T.Lee ${ }^{\dagger}$ \\ *School of Electronics \& Computer Science, University of Southampton, SO17 1BJ, UK \\ †Mesophotonics Ltd, 2 Venture Rd, Chilworth Science Park, SO16 7NP, UK
}

\begin{abstract}
In this paper we investigate improvement in performance attainable by etching Photonic Crystals and Photonic Quasi-Crystals into the top emitting surface of LEDs. We describe the physical mechanisms of extraction enhancement through ordered surface patterning and investigate benefits in terms of total extraction enhancement, beam directionality, and far field beam quality. Factors such as lattice geometry, etch depth, and epitaxy thickness are investigated. We show that a great variety of far field beam profiles of benefit in applications such as projection TV light engines and direct flat panel display illumination can be obtained simply by adjusting geometric design parameters. Our results show that PCs can provide significant improvement in extraction enhancement for applications requiring non Lambertian beam shapes when etched into standard "production line" epitaxy wafers in comparison to "state of the art" surface roughened thin-GaN LED devices. We investigate PC beam steering effects in these devices confirming that PCs do in fact re-direct light from trapped modes confined within the epi-structure to radiating modes. We also show that by tailoring the thickness of the epi-structure to complement the properties of the photonic crystal, extraction enhancement can be improved by a factor of 9 for some applications.
\end{abstract}

Keywords: Photonic Crystal, LED, Quasicrystals, extraction enhancement.

\section{IMPROVING LED EXTRACTION EFFICIENCY THROUGH SURFACE PATTERNING}

Typical surface emitting LEDs consist of a several micron thick high refractive index GaN slab layer with embedded Multiple Quantum Wells (MQWs) positioned some distance from the surface ${ }^{1}$. In a conventional unpatterned surface emitting LED the majority of light emitted from the quantum wells become trapped in the high index GaN slab layer by a process of total internal reflection (TIR) at the $\mathrm{GaN}$ air and $\mathrm{GaN} /$ substrate boundaries, in which case emitted photons become confined to a multitude of confined slab waveguide modes. Since only a small fraction (about $4.35 \%$ ) of emitted light radiates away from the top surface to free space, bare unroughened LED devices suffer from issues of poor wall-plug efficiency ${ }^{2}$.

The physical process of confinement can be explained diagrammatically by the Energy-K vector (E-K) diagram (fig 1a). In this diagram, the $\mathrm{y}$-axis (energy) relates to the free space wavelength of emitted light, and the $\mathrm{x}$-axis ( $\mathrm{k}$ vector) relates to azimuth angle of emission from the quantum wells, and the wavelength of emitted light whilst propagating within the device. The solid lines (dispersion curves) indicate the permitted energy states for emitted light. The dashed line indicates the "light line" which represents the angular condition for TIR. Modes lying below the light line will be confined within the LED GaN layer by TIR, whereas those lying in the shaded region above the light line are free to radiate to free space. In reality LEDs emit over a narrow range of wavelengths (about $25 \mathrm{~nm}$ ), which can be approximated on the E-K diagram by a horizontal (dashed) sample wavelength line. Points of intersection between the sample wavelength line and the dispersion curves then indicate the permitted modes of emission for the LED structure. From the diagram we can see that for an unpatterned LED, the majority of the modes lie below the light line and so are strongly trapped within the GaN slab.

Introduction of periodic patterning on top of the LED causes the dispersion curves to become folded at the Brillouin zone boundary (represented by the vertical dashed lines in fig 1a). In this case the sample wavelength line then intersects not only a number of trapped modes lying below the light line, but also a set of band-folded Photonic Crystal-

Light-Emitting Diodes: Research, Manufacturing, and Applications XI, edited by Klaus P. Streubel, Heonsu Jeon, Proc. of SPIE Vol. 6486, 64860R, (2007) - 0277-786X/07/\$18 - doi: 10.1117/12.715025 
Bloch modes now lying above the light line. In this case scattering events within the structure (originating from multiple reflections of photons from the dielectric interfaces of the holes etched into the surface) allow emitted photons to jump freely between confined and radiating modes. Hence by etching a photonic crystal some distance into the top of a conventional LED structure, an "engineered" leakage mechanism can be introduced into the structure which can significantly improve extraction efficiency. Geometric factors such as lattice shape, hole size, and dielectric constants of materials used play an important role in determining the efficiency and directionality of the resultant leaky modes. Two key factors affecting the performance of PC LED devices are etch depth and lattice symmetry. These factors will now be explored in detail.

\section{Energy}

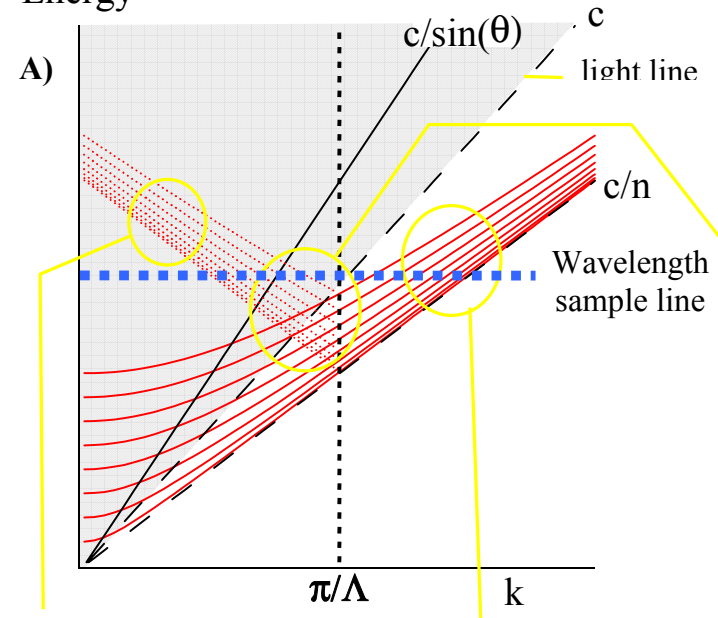

Radiating PC Bloch modes (above light line)

B)

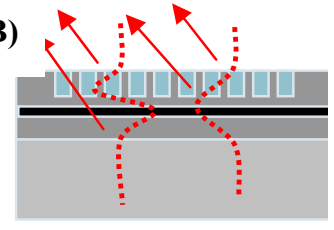

Confined slab modes (below light line)

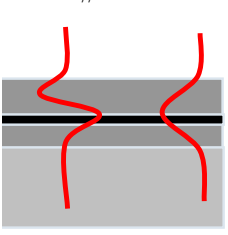

D)

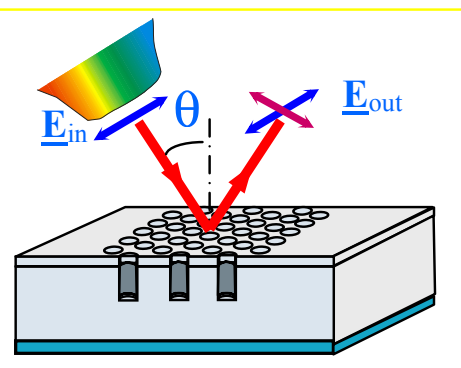

C)

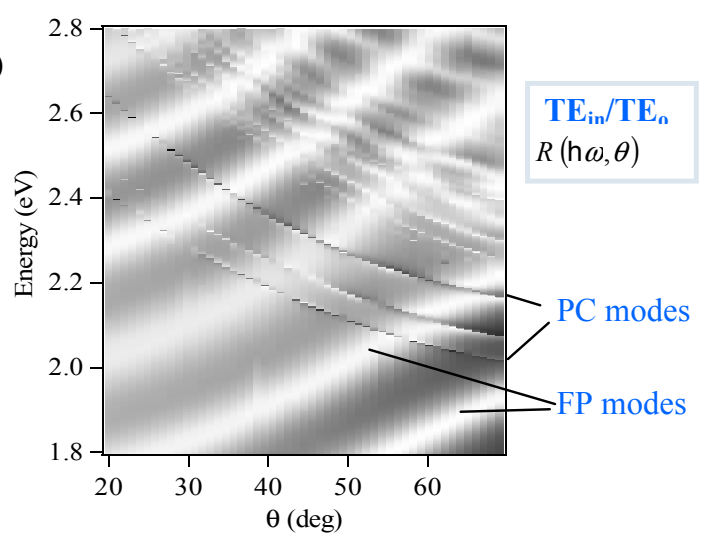

Figure 1: a) Energy K-vector diagram for a PC-LED device. Solid lines - confined slab modes, dashed lines, band folded PC Bloch modes. Shaded area - radiating modes above light line, vertical dashed line - Brillouin zone boundary. b) representation of corresponding mode profiles in LED layer structure. c) experimental mapping of PC slab device dispersion by spectral reflectometry. d) diagrammatic representation of experimental setup

\section{Effect of etch depth}

Etch depth plays an important role in determining the efficiency of cross-coupling between trapped waveguide modes associated with the thick slab structure and leaky Bloch modes associated with the two dimensional surface patterning. Typical LEDs support over 50 trapped slab waveguide modes. For maximum extraction efficiency there must be strong coupling between trapped slab waveguide modes and leaky PC Bloch modes. Shallow etched PC structures only allow cross coupling between a few higher order trapped slab modes whereas PCs etched within close proximity to the MQWs allow extraction of nearly all the trapped slab modes. Due to practical problems involved in etching very deep holes in GaN, PCs will work inherently better with thin LEDs.

The complex coupling processes linking confined slab modes and leaky PC Bloch modes can be explored experimentally by spectral reflectometry ${ }^{3}$ (fig 1b). In this experiment a broadband laser is directed onto the surface of a 
thick Slab PC structure at a well defined azimuth angle $(\theta)$ and the spectra of the specular reflection taken at the same angle using a fiber coupled spectrometer. At certain wavelengths and azimuth angles, incident light will partially scatter into confined modes by the reverse coupling process that would occur during emission of photons in an LED, resulting in a subsequent reduction in reflected power. By scanning azimuth angle and wavelength, the dispersion relations of the structure can then be mapped by tracking dips in reflectivity. In fig $1 \mathrm{~b}$ the broad diagonal lines sloping from bottom left to top right correspond to trapped slab waveguide modes. However, narrower lines cross these lines sloping from right to left of the diagrams. These features represent leaky Block modes associated with the periodic surface patterning. In the case of two-dimensional surface patterning these features become quite complex illustrating the complexity of cross coupling of photons between confined slab waveguide modes and leaky Bloch mode of the Photonic crystal lattice. In effect his experiment maps out the predicted extracted modes of an LED for all wavelengths of light.

\section{Effect of lattice symmetry}

Photonic crystals have traditionally emulated natural periodic structures such as gratings and square or triangular lattices which respectively have 2, 4 and 6 fold symmetries. As the level of symmetry increases the properties of the lattice become less directional. The highest degree of symmetry found in nature is given by the triangular lattice which has 6-fold symmetry.

A)

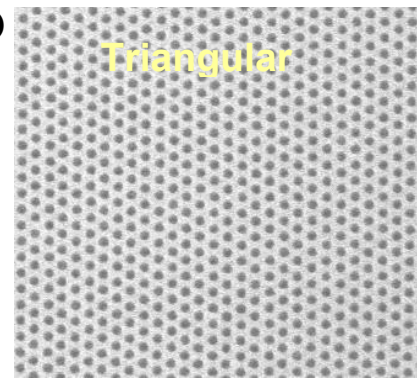

D)

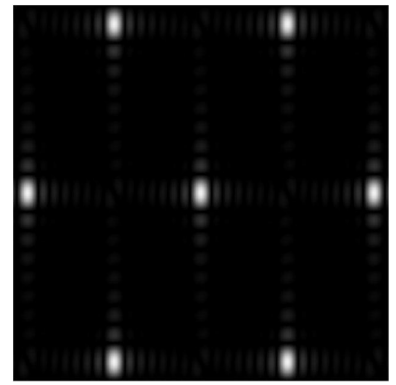

F)

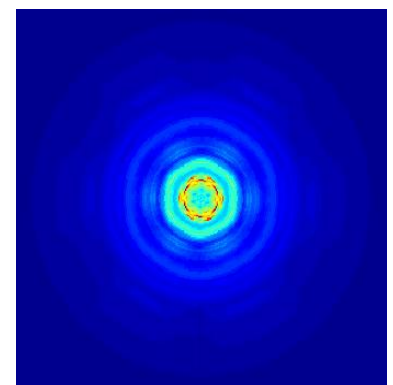

B)

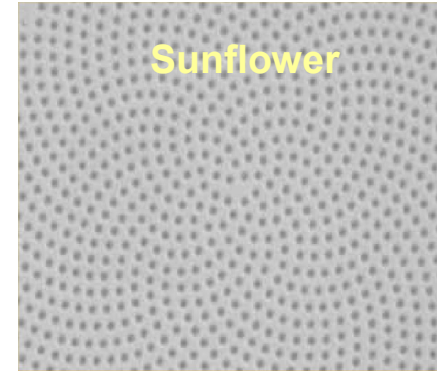

E)

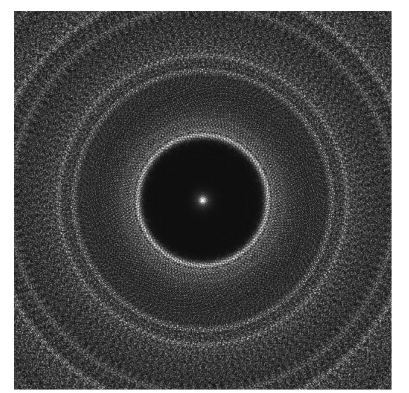

G)

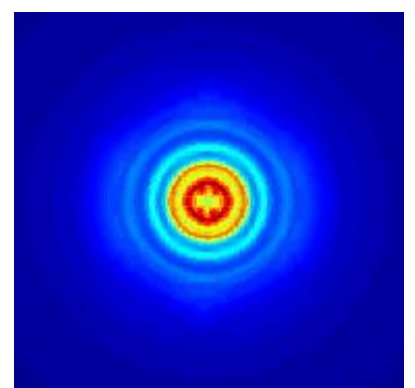

C)

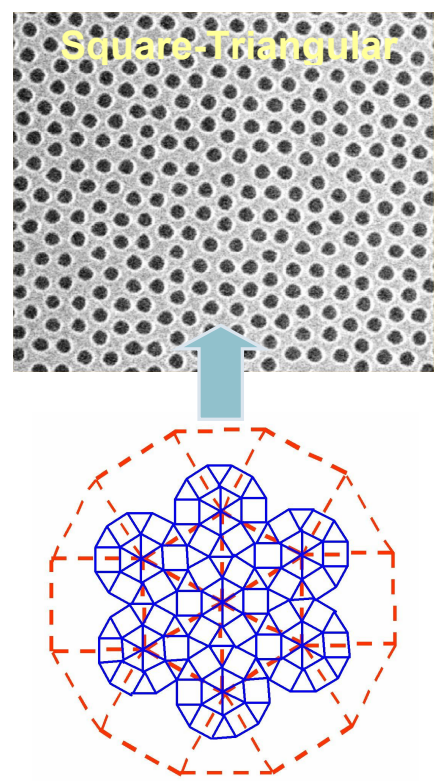

Figure 2: Various $\mathrm{PC}$ and $\mathrm{PQC}$ lattice tiling schemes $(\mathrm{A}, \mathrm{B}, \mathrm{C})$ and their corresponding far-field beam profiles $(\mathrm{F}, \mathrm{G})$ and diffraction patterns $(\mathrm{D}, \mathrm{E})$. 


\section{INTRODUCTION TO PHOTONIC CRYSTALS AND QUASICRYSTALS}

Since photonic crystals are artificial photolithography defined structures, lattice patterns need not necessarily be constrained to designs found in nature. A completely different class of structures known as Photonic Quasi-Crystals exist. These (PQC) are unusual in the respect that on first sight they appear random, however on closer inspection reveal themselves to possess long range order but short range disorder ${ }^{4}$. Some example PQC structures are shown in fig 2. Square-triangular lattice (fig 2c) is grown by use of the random Stampfli inflation method. This provides an approximation to a dodecagonal quasi-crystal. The recursive tiling of offspring dodecagons packed with random ensembles of squares and triangles in dilated parent cells forms the lattice. This class of PQC possess orders of symmetry relating to the primitive cell shape. The sunflower lattice (fig $\mathbf{2 b}$ ) is constructed from the Fibranacci series, and possess maximal packing of rods around a central region of space.

Analysis of the far field diffraction pattern (Fourier transform (FT)) of a lattice structure reveals the true nature of its symmetry. Regular lattices (and those with finite levels of symmetry such as square-triangular, Penrose and Archimedean tiling ${ }^{\mathbf{5}}$ ) reveal their symmetry by way of distinctly defined Bragg-spots (fig2d). The far field transform of the Fibronacci lattice (fig 2e) however reveals a well defined circular ring with no such artefacts in space surrounding the central Bragg spot. This lattice shows near perfect circular symmetry, and thus would be expected to have extremely isotropic dispersion properties. In the context of LED light extraction, the far field pattern of a PC-LED would be expected to show structural artifacts similar to that of the far-field diffraction pattern but less pronounced due to the fact that k-vectors effectively become integrated by an observer over a finite angular range. Simulated PC-LED beam patterns are shown in (fig $\mathbf{2} \mathbf{f , g}$ ).

\section{MODELLING OF FLIP-CHIP THIN GAN LED DEVICES BY FDTD.}

In order to investigate the properties of PQCs, Finite Difference Time Domain (FDTD) methods were adopted. In order to obtain realistic simulation results it is important to model structures which approximate closely to real operational designs. In modeling PC-LED devices emission dipole characteristics were simulated as closely as possible to those in a real LED by locating a multitude of dipoles (emitting randomly in time) across a single QW placed some distance below the top patterned surface of the LED. To run a realistic simulation, a relatively large percentage of an LED die area must be modeled. (typically 25 microns width were used). Distributed computing techniques were employed to overcome issues of long run times and fine grid meshes, utilizing a 128 dual node dual core AMD processor cluster with $2 \mathrm{~GB}$ of memory per core. Each simulation returned not only total extraction efficiency, but full information about far field beam patterns and beam steering efficiency of the PC.

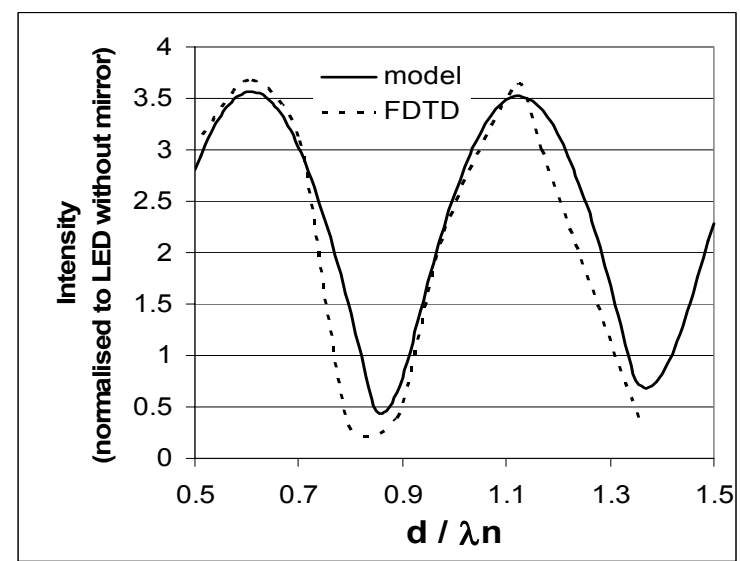

Figure 3: Comparison of FDTD and analytical models for cavity-enhanced thin GaN LED designs.

Currently there is great interest in thin GaN flip chip LEDs which utilize cavity effects for improved extraction efficiency $^{6}$. These devices incorporate a reflective layer placed a small distance behind the Quantum Wells Such that interference effects distort the shape of the far field emission profile so that more energy is directed into the forward 
emission direction. These devices can be successfully described by a simple analytical model ${ }^{6}$ which has been validated against experimental measurements on fabricated devices. Consequently, conventional unpatterned flip chip GaN LEDs provide a good reference design to validate our FDTD model. Fig 3 shows extraction efficiency as a function of distance between the mirror and quantum wells, calculated using both FDTD and conventional analytical models. Good agreement was found between the two methods, although small discrepancies exist due to the fact that a discrete mesh is utilized in FDTD which will not precisely resolve amplitude at resonance. Ultimately, both methods confirm that if properly tuned, a factor of $1.75 \mathrm{X}$ improvement can be obtained in comparison to a standard LED with a perfect lossless mirror placed some distance behind the quantum wells. Furthermore simulated beam profiles generated by flip chip LEDs using the FDTD method match well with published profiles for real fabricated flip-chip GaN LEDs.

\section{IMPROVEMENTS IN EXTRACTION EFFICIENCY}

As a first step towards commercialization of PC-LEDs it is desirable to incorporate PCs into current conventional LED epi-structures. As has been mentioned earlier, the existence of more than 50 waveguide modes in typical thin-GaN devices does not allow PCs to operate optimally. Nonetheless we now present numerical extraction results comparing both roughened and PC devices on conventional epi and recap on improvement achievable by design features such as mirrors, cavity effects roughening and PCs. Since flip-chip thin-GaN designs are the most efficient currently available devices, these will be used as the example device platform.

Incorporation of a perfect mirror in a thin GaN LED gives a maximum theoretical improvement in total extraction efficiency (in comparison to a standard Lambertian source) of x2 (this assumes that the mirror collects over all angles and is loss-less). Inclusion of a cavity-interference design (as described in the previous section for validation of our FDTD model), gives a further improvement factor of X1.75 over an LED incorporating a perfect mirror.

In order to increase the light extraction from conventional LEDs scattering centers are usually introduced into the top surface of the $\mathrm{n}-\mathrm{GaN}$. State of the art roughening techniques employ wet etching of the $\mathrm{n}-\mathrm{GaN}$ to form pyramid structures $^{7,8,9,10}$. This was modeled using randomly sized pyramids in the region of $0.5-1 \mu \mathrm{m}$ and was found to provide a further factor of x 2.5 improvement in total light extraction.

Inclusion of a Photonic crystal in an unoptimised thin-GaN epitaxy design (GaN thickness $\sim 5 \mu$ m) was found to provide a factor of X2.5 improvement in total extraction efficiency which is very similar to what can be obtained with "state of the art" surface roughening. However, looking at extraction into a reduced cone half angle of $30^{\circ}$ we find that PCs can provide an improvement factor of X1.6 for a microcavity LED design in comparison to a conventional Lambertian device.

In conclusion, PCs cannot out-perform the very best surface roughened LEDs in terms of total extraction efficiency, however they can provide very significant improvements in performance in terms of redirection of emitted light into a directional beam, which provides significant benefits in many practical applications. They can also provide improvements in power output yield of LED die due to better reproducibility of the PC patterns on the surface. Directional beam-shaping properties of PC-LEDs will now be explored in more detail.

\section{PC-LED BEAMSHAPING EFFECTS}

Choice of PC geometry plays an important part in determining the far field emission pattern of an LED allowing profiles to be tailored to the requirements of specific applications. For example, PCs allow the creation of nonLambertian sources (without secondary expensive microlensed packaging), and tailored illumination of the far-field, leading to improved efficiency for a given application (by ensuring that more light is coupled from the source to the application), more efficient lower cost packaging and more efficient thermal management. Perhaps most significantly PCs allow a single epistructure to become suitable for a multitude of applications, whereby a change of application requires adjustment of the PC lattice geometry rather than the entire epi-structure of the wafer.

Fig 4 shows simulated beam profiles for a variety of photonic crystal and PQC structures etched into thin GaN devices (with optimized epi-layers). Dependent upon the combination of PC or PQC design parameters a variety of beam profiles can be produced. Profiles to the left would be good for projection display applications (tighter light cone),

and those to the right, direct flat panel display illumination where it is desirable to emit light into side lobes. This figure clearly highlights the diverse nature of the far field patterns achievable in both cases. 


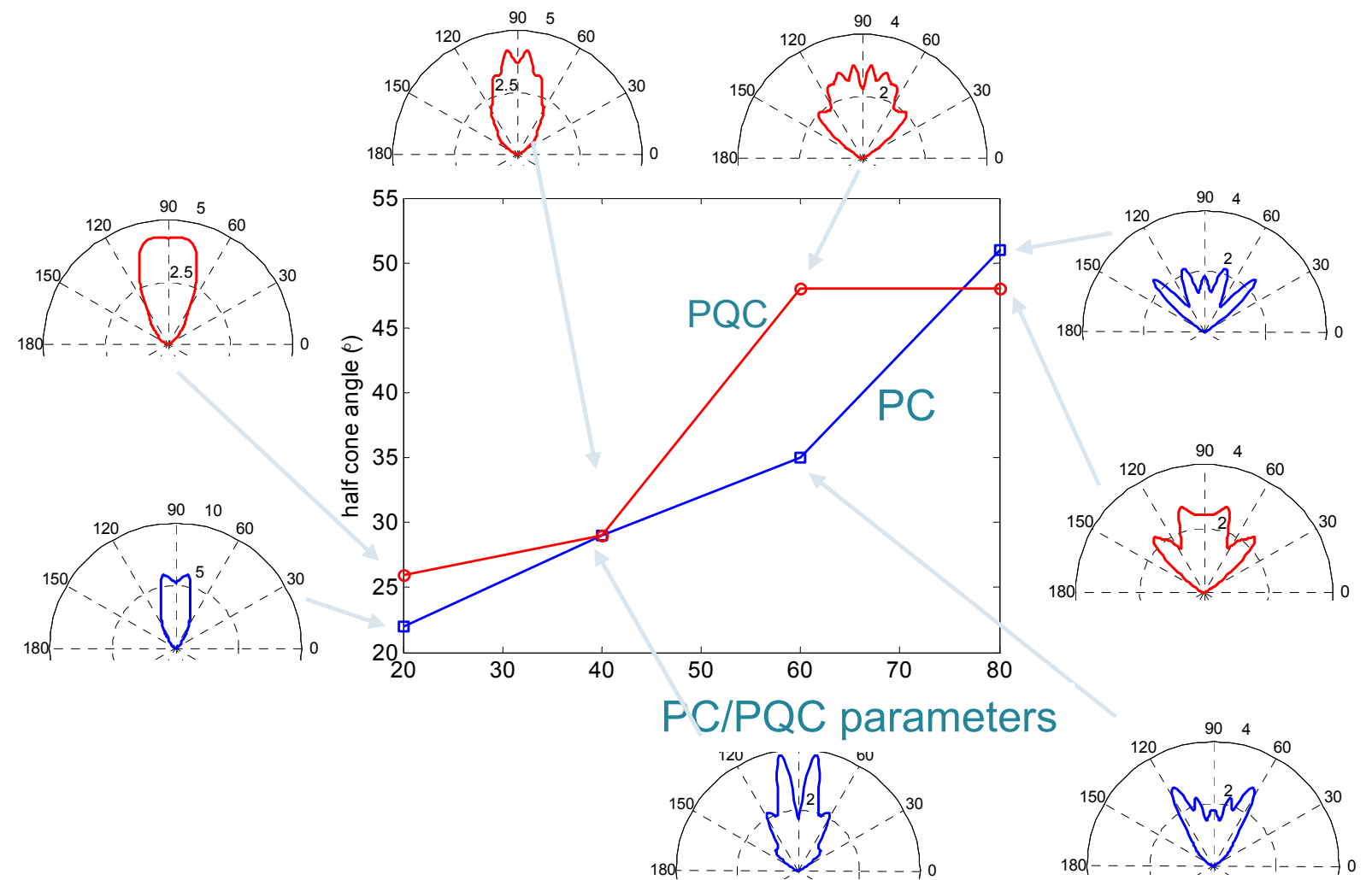

Figure 4: Variety of beam profiles which can be obtained using PC structures.

Although total extraction efficiencies for regular PCs and PQCs can be quite similar, far field emission patterns for regular PCs can show highly structured artifacts which can be problematic for applications. Fig 5 shows a comparison between the radial properties of the emission pattern for a square lattice and PQC-PC. The PQC has a far more isotropic beam profile which in the context of applications would remove the need for secondary optics (for projection displays) and eliminates the need for complex diffusers for backlight displays. Lattice symmetry not only has an important effect on extraction efficiency and beam directionality but also on the quality of the far field emission pattern as shown in fig $2 \mathrm{~g}, \mathrm{~h}$.

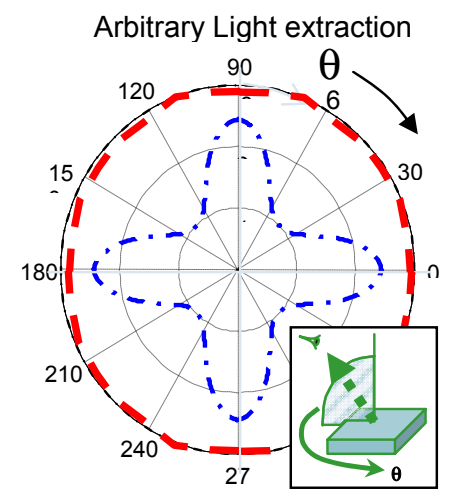

Figure 5: Comparison between radial properties of a regular Photonic Crystal and Photonic Quasi-crystal LED design. 


\section{FURTHER IMPORVEMENTS THROUGH OPTIMISATION OF EPI-STRUCTURE}

Fig 6a shows the beam profile for a Photonic Crystal etched into a standard epitaxy design. The solid line indicates Lambertian source emission, dashed-dot line indicates emission profile from a side emitting LED module, dotted line shows emission for a PC-LED device. The PC design projects light predominantly into two side lobes. (This non Lambertian beam profile would be useful for direct flat panel display illumination). We find that the maximum intensity of the side lobes is approximately three times that of a standard Lambertian, whereas current side emitting LED module give a maximum intensity equivalent to the Lambertian source.

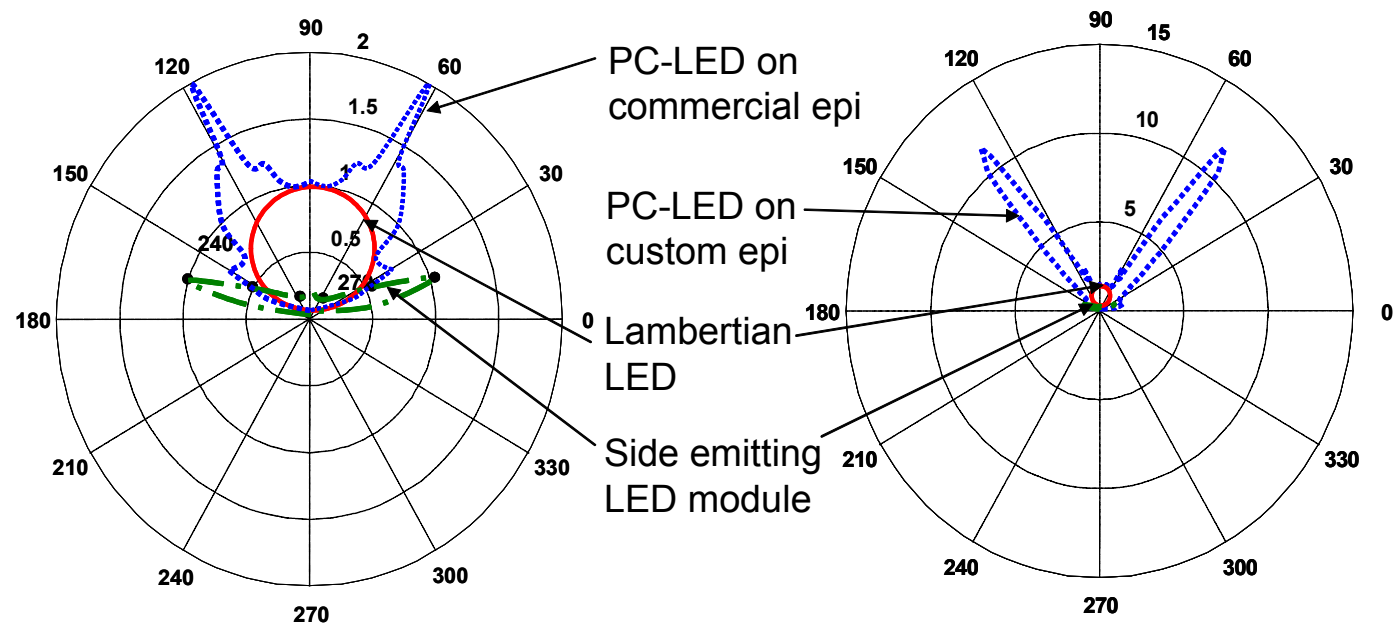

Figure 6: Emission profiles for (A) standard-epi design and (B) tailored-epi. (Solid line - bare unroughened Lambertian device, dotted line - PC device, dash-dot line - side emitting LED module).

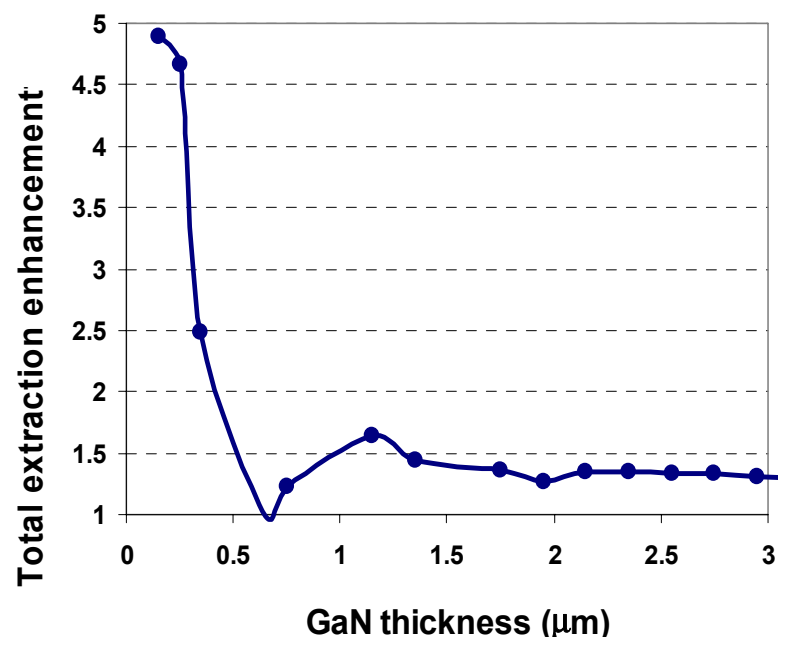

Figure 7: Effect of epi layer thickness on total extraction enhancement in comparison to a bare unroughened Lambertian LED. 
Fig $6 \mathbf{b}$ shows the improvement which can be obtained by tailoring the thickness of the epi-structure to complement the photonic crystal design. In this case the side lobes are far better defined (fewer structural artifacts) and extend over a much narrower angular range. Most significantly extraction efficiency into this narrow cone angle is improved by a factor of nearly $\mathrm{x} 9$ compared to the same angular range of emission from a standard Lambertian source. The projection angle of the side lobes can be tailored by adjusting the PC design parameters. These effects would be most beneficial for direct illumination of thin flat panel or flexible displays where optimal illumination can be obtained by matching emission angle to the thickness of the display panel.

fig 7 shows total extraction enhancement (integrated over all angles) as a function of epi-layer thickness in comparison to a standard bare unroughened Lambertian LED. In this case total extraction enhancement can be improved by a factor of X5 compared to a standard Lambertian by optimizing the thickness of the epi-layer to complement the PC design. Most significant gains are obtained for epi-layer thickness between 300-500nm where the PC interacts with one or two trapped waveguide modes.

\section{ANALYSIS OF PHOTONIC CRYSTAL BEAM STEARING EFFECTS IN LEDs:}

There is some debate within the LED community as to whether a Photonic Crystal etched on top of an LED device really do redirect trapped light with the $\mathrm{GaN}$ to radiative modes thereby improving extraction efficiency, or instead act as a filter device simply reflecting light incident upon the surface of the LED at angles outside a narrow extraction cone back into the GaN layer which would not improve extraction efficiency.

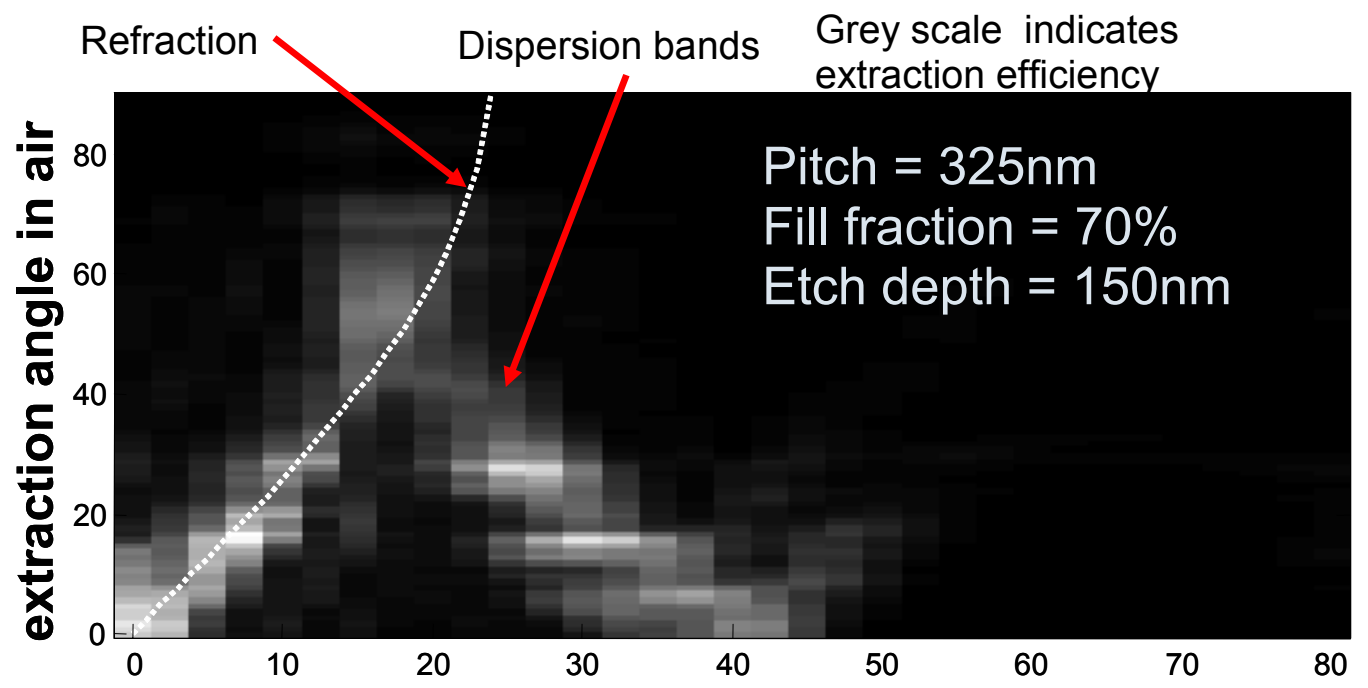

Figure 8: Beam steering properties of a Photonic Crystal etched into the top surface of an LED.

To answer this question, we investigate extraction angle as a function of angle of emission from the underlying quantum wells (fig 8) inside the GaN material. Superimposed upon fig 8 is the expected trend due to refraction calculated at the GaN -air interface using Snells law.

The data in fig 8 shows that for rays emitted near vertically out of the top of the PC-LED (eg along the axis of the holes or the normal to the surface), the PC-LED does indeed act predominantly as a refracting medium. However at angles greater than the critical angle for TIR the PC does indeed re-direct light to much shallower emission angles. For example: rays incident to the surface of the $\mathrm{GaN}$ at $40^{\circ}$ with respect to the normal to the surface actually radiate into air and propagate almost along the normal direction $\left(0^{\circ}\right)$. In fact as incidence angle increases beyond $18^{0}$, the free space extraction angle becomes progressively reduced. This effect cannot be explained by simple refraction alone. Hence this data shows that PCs really do scatter rays emitted at angles greater than the critical angle for TIR to radiative modes in air at angles less than those proposed by refraction via strong beam steering effects not associated with refraction.. 
From a physical perspective this is not unexpected since rays propagating near perpendicular to a shallow etched PC will have a very small in-plane wave-vector component, which means that the interaction of photons propagating at this angle within the GaN will be weak as there is limited possibility for scattering in the lateral plane between holes during their short transit through the photonic crystal layer. This in turn results in weak coupling between trapped slab waveguide modes and radiative Bloch modes of the photonic crystal.

However, photons emitted at larger angles (20-50 deg), will have larger in-plane wave-vector component, and therefore interact more strongly with the periodic structure of the PC by scattering in the lateral direction during their transit through the device, resulting in stronger coupling between trapped waveguide modes and Bloch modes of the $P C$.

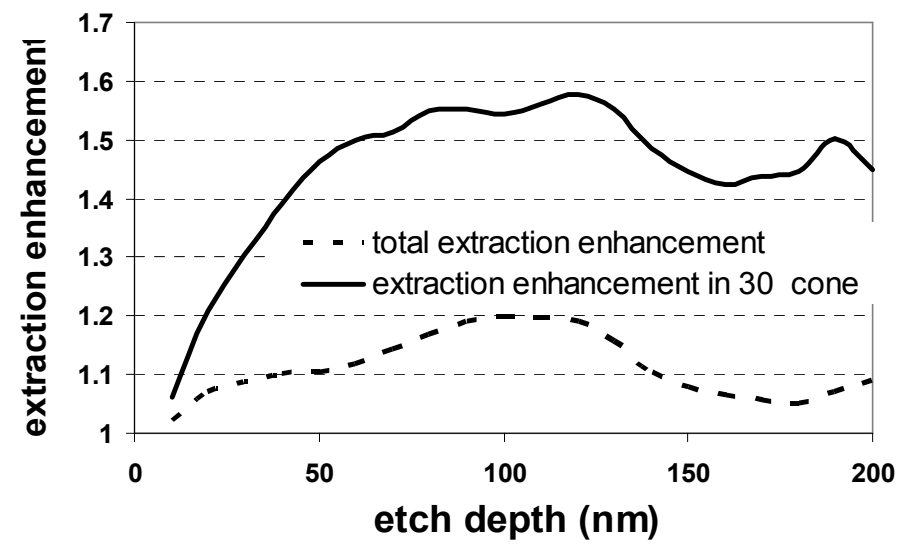

Figure 9: Effect of PC etch depth on extraction efficiency

Looking at the effect of etch depth in more detail, as etch depth increases less energy becomes extracted through refraction and more by photonic crystal beam-steering effects. Fig 9 shows a comparison between total extraction enhancement and extraction enhancement into a $30^{\circ}$ cone as a function of etch depth. There actually turns out to be an optimal etch depth of 120nm for enhanced extraction efficiency in both cases. Etching deeper introduces reflection effects between lower refractive index PC region and the underlying GaN and hence an optimized PC etch depth is required to achieve an anti-reflection coating type effect.

\section{CONCLUSIONS}

In conclusion we have discussed several factors relating to the design of PC-LEDs and their effect on LED extraction enhancement and beam profiles. These include: effect of lattice symmetry, and etch depth. Benefits of unusual lattice structures known as Photonic QuasiCrystals were presented and quantitive comparisons were drawn with more conventional methods of improving extraction efficiency such as random surface roughening, and utilisation of mirrors and microcavity effects. Although PCs do not appear to improve total extraction efficiency for applications requiring a Lambertian source beyond what is achievable by current surface roughening techniques, when incorporated in current epistructure wafers, there are potential benefits in terms of improved yield across a wafer and beam directionality. In relation to beamshaping PCs were shown to provide a $60 \%$ improvement in extraction efficiency into a $30^{\circ}$ half cone angle. Properties of the beam profile can be modified by adjusting geometric parameters such that a single epitaxy design can be utilised for a variety of different applications. However, most significant gains can be achieved by optimising the epitaxy design to complement the photonic crystal, in which case an improvement in extraction efficiency for applications such as direct flat panel illumination of $900 \%$ over current bare unroughened Lambertian devices can theoretically be obtained. 


\section{REFERENCES}

${ }^{1}$ EF Shubert "Light emitting diodes" Cambridge U press, 2003

${ }^{2}$ I. Schnitzer, E. Yablonovitch, C. Caneau, A.Scherer, Appl Phys Lett. 63 (16) 1993

${ }^{3}$ M.C.Netti, A.Harris., J.J.Baumberg., D.Whittaker., M.D.B.Charlton., M.E.Zoorob., J.S.Wilkinson \& G.J.Parker., Phys Rev Letts, 2001, vol 86, p1525

${ }^{4}$ Zoorob, M.E.; Charlton, M.D.B.; Parker, G.J.; Baumberg, J.J.; Netti, M.C. Nature, v 404, n 6779, 13 April 2000, p 740-3

${ }^{5}$ M.Rattier, H.Benisty, E.Schwoob, C.Weisbuch, T.F.Krauss, C.J.M.Smith, R.Houdre, U.Oesterle, App Phys Letts, 837 (2003).

${ }^{6}$ Shen et al, Appl. Phys. Lett. 82, (14) (2003).

${ }^{7}$ W.C. Peng, Y.C.S. Wo,Appl Phys Letts 88 (2006)

${ }^{8}$ C.Huh, K.S.Lee, E.J.Kang, S.J.Park, J.Appl.Phys, 93, 11 (2003)

${ }^{9}$ H.W.Huang, J.T.Chu, C.C.Kao, T.H.Hseuh, T.C.Lu, H.C.Kuo, S.C.Wang, C.C.Yu, Nanotechnology 16 (2005)

${ }^{10}$ T.Fujii, Y.Gao, R.Sharma, E.L.Hu, S.P.DenBaars, S.Nakamura, App Phys. Letts, 846 (2004) 